\title{
Using Microfluidic Chips with Nanochannels for Measuring the Mean Inner Potential of Liquid Water by Off-Axis Electron Holography
}

\author{
Murat Nulati Yesibolati ${ }^{1}$, Simone Laganà ${ }^{1}$, Takeshi Kasama ${ }^{2}$, Hongyu Sun $^{1}$, Silvia Canepa ${ }^{1}$ \\ and Kristian Mølhave ${ }^{1}$
}

${ }^{1 .}$ Building 345E, Department of Micro- and Nanotechnology, Technical University of Denmark, 2800 Kongens Lyngby, Denmark.

2. DTU Danchip, Center for Electron Nanoscopy, Technical University of Denmark, Fysikvej, 2800, Kongens Lyngby, Denmark.

Electrons interact with the electric and magnetic fields existing within and around a TEM specimen which leads to changes in the transmitted electron wave function. Electron holography [1] can be used to measure the phase and amplitude changes of the electron wave. Off- axis electron holography[2] requires a coherent and bright beam split into two parts: one passes through sample as object wave; one pass through vacuum as reference wave. A charged biprism wire tilts the reference wave relative to the object wave, so that the two waves interfere and a hologram TEM image can be recorded.

The mean inner potential (MIP) $\mathrm{V}_{0}$ is a volume-averaged electrostatic potential of a material [3, 4]. It is a fundamental property of the material, and depends on both composition and structure. It is useful for e.g. studies of the work function, iconicity of the solid, band structure in van der Waals bonded crystals. Electron holography is potentially the most accurate method to measure MIP, and considerable efforts have been focused on determining the MIP for solid materials mostly with known geometries [5].

However, very little has been made on liquid phase materials with MIP measurement until now. This is due to the limitation by several factors: Firstly, water based solutions have high vapor pressures making them technically difficult to introduce into the high vacuum in a TEM, and they are susceptible to charging and beam damage under electron beams. Secondly, electron holographic MIP measurement requires accurate geometry and thickness measurements [6], which can be difficult to achieve on liquid samples. So far, work has only been done on vitrified ice [7]. Ionic liquid (IL) has also been studied, but found that electron irradiation changed the electrostatic potential around the IL, and specific MIP value was not obtained [8].

The change in phase $\Delta \varphi$ of an electron wave passing through a material relative to the phase of an electron wave passing through vacuum, is proportional to both thickness $t$ and the $\mathrm{MIP}_{\mathrm{o}}$ as written equation below [9]:

$$
\Delta \varphi=\frac{2 \pi}{\lambda} \frac{E_{0}+E}{2 E_{0}+E} V_{0} t=C_{E} V_{0} t
$$

Where $\lambda$ is the electron wavelength, e is the electron charge, $\mathrm{E}$ is the electron kinetic energy, and $\mathrm{E}_{0}$ is the electron rest energy. The first term can be simplified as $\mathrm{C}_{\mathrm{E}}$, a constant depending only on the electron beam energy that equals $6.53 \times 10^{6} \mathrm{rad} / \mathrm{Vm}$ at $\mathrm{E}=300 \mathrm{keV}$. Hence, if both the phase change $\Delta \varphi$ relative to the vacuum and the thickness $\mathrm{t}$ are known in a liquid sample, the MIP of that sample can be calculated straightforwardly. 
Here, we take advantage of the recent development of in-situ liquid TEM microchip systems used to observe processes in aqueous or other liquid samples [10] and use a newly developed system to measure the MIP of liquid water solution of $1 \mathrm{mM} \mathrm{NaCl}$. Multiple suspended $\mathrm{Si}_{3} \mathrm{~N}_{4}$ nanochannels for TEM were created by bonding two silicon microchips. More details about the chip fabrication were reported in our previous work [11]. As illustrated in Figure 1A, the liquid solution is confined in an electron transparent $\mathrm{Si}_{3} \mathrm{~N}_{4}$ nanochannel, which is supported by two bonding $\mathrm{Si}_{3} \mathrm{~N}_{4}$ membranes. The nanochannel height, namely the liquid thickness was determined already from both the fabrication process and EELS measurements to be $100 \pm 3 \mathrm{~nm}$. The phase image in Figure $1 \mathrm{~B}$ was reconstructed from the original hologram images. A phase change of $2.1 \pm 0.4 \mathrm{rad}$ was found from the liquid sample and suspended $\mathrm{Si}_{3} \mathrm{~N}_{4}$ membranes relative to the enclosing $\mathrm{Si}_{3} \mathrm{~N}_{4}$ membranes. Therefore, using the equation above, the MIP $\mathrm{V}_{0}$ of water solution is calculated to be $3.2 \pm 0.36 \mathrm{~V}$ which within error matches the published value of $3.5 \pm 1.2 \mathrm{~V}$ measured on vitrified ice [12].

\section{References:}

[1] D Gabor, Nature 161 (1948), p. 777.

[2] G Mollenstedt and H Duker, Zeitschrift Fur Physik 145 (1956), p. 377.

[3] H Bethe, Annalen der Physik 87 (1928), p. 55.

[4] A Sanchez and MA Ochando, Journal of Physics C-Solid State Physics 18 (1985), p. 33.

[5] T Tanigaki et al, Journal of Physics D-Applied Physics 49 (2016).

[6] M Gajdardziskajosifovska et al, Ultramicroscopy 50 (1993), p. 285.

[7] A Harsher and H Lichte, Electron Microscopy 1 (1998), p. 553.

[8] M Shirai et al, Ultramicroscopy 146 (2014), p. 125.

[9] LFA Edgar Völkl and CJ David in "Introduction to Electron Holography", (Springer US) p. 354.

[10] FM Ross, Science 350 (2015).

[11] S Lagana et al, European Microscopy Congress (EMC) 2016, DOI: 10.1002/emc2016.0319.

[12] We would like to thank DTU Nanotech, DTU CEN, DTU Danchip, and the funding from the

Danish Research Council for Technology and Production Case No. 12-126194.
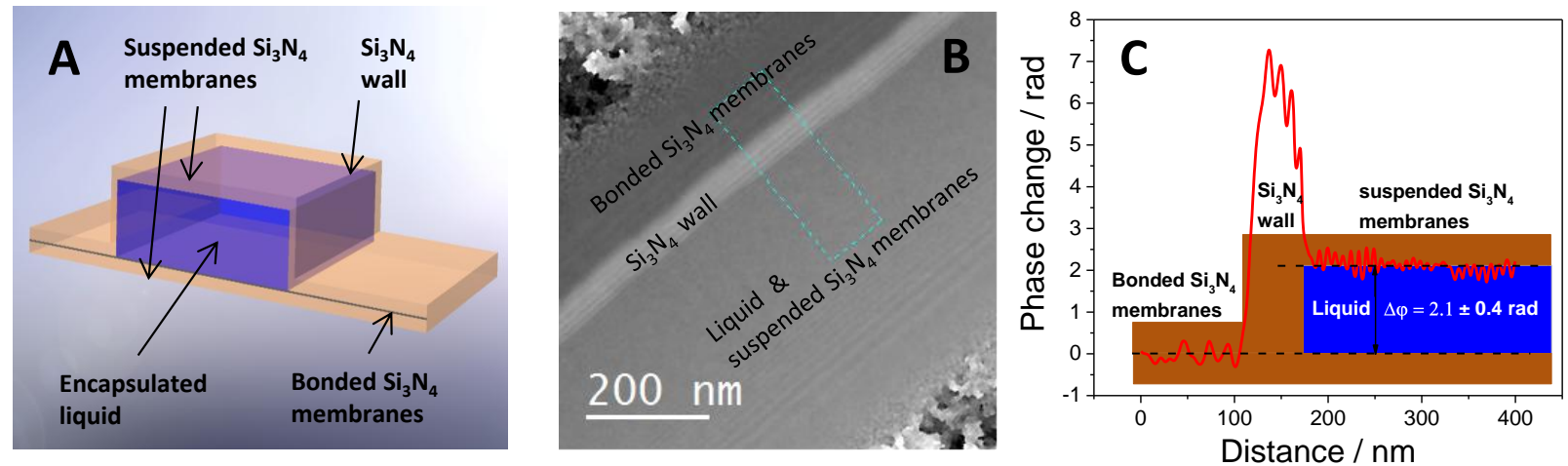

Figure 1. A). A schematic illustration of a single nanochannel filled with liquid sample of $1 \mathrm{mM} \mathrm{NaCl}$ water solution; B). Reconstructed phase image from original hologram images; C). A phase profile from Figure $1 \mathrm{~B}$ obtained by averaging the phase image over $110 \mathrm{~nm}$ parallel to the $\mathrm{Si}_{3} \mathrm{~N}_{4}$ wall. 